\title{
REPRODUCIBLE MODEL FOR KIDNEY AUTOTRANSPLANTATION IN PIGS
}

\author{
I. Jochmans ${ }^{1}$, E. Lerut ${ }^{2}$, V. Heedfeld ${ }^{1}$, T. Wylin ${ }^{1}$, J. Pirenne ${ }^{1}$ and D. Monbaliu ${ }^{1}$
}
${ }^{1}$ Abdominal Transplant Surgery, Department of Surgery, Catholic University of Leuven, Leuven, Belgium

${ }^{2}$ Department of Morphology and Molecular Pathology, University Hospitals Leuven, Leuven, Belgium

Ina Jochmans is associated with the Research Foundation - Flanders, Belgium (FWO Aspirant)

This study was supported by an unrestricted grant from Astellas Pharma Inc.

Jacques Pirenne and Diethard Monbaliu are holders of the chair "Abdominal Transplant Surgery from the Centrale Afdeling voor Fractionering”, Vilvoorde, Belgium.

Address reprint requests to Ina Jochmans, Abdominal Transplant Surgery, Department of Surgery, Catholic University of Leuven, Herestraat 49, 3000 Leuven, Belgium.

Tel. +32 $16348727 \quad$ Fax.+3216348743 E-mail: ina.jochmans@med.kuleuven.be

\begin{abstract}
Introduction. Several porcine models to study mechanisms of warm ischemia, cold ischemia and ischemia-reperfusion injury exist, but the technical and surgical aspects of these models and their possible pitfalls have not been described in detail. The goal of the present study was to develop a porcine kidney autotransplantation model, to report the difficulties encountered and possible solutions.
\end{abstract} Methods. Twelve female pigs (24-51 kg) underwent a left ureteronephrectomy. The procured kidney was flushed with $500 \mathrm{~mL}$ of histidine-tryptophan-ketoglutarate preservation solution and subsequently cold stored in University of Wisconsin preservation solution. An autotransplantation was performed 18 hours later, following a contralateral nephrectomy. Serum creatinine and urine production were assessed post-transplantation. Pigs were sacrificed ten days post-transplant.

Results. Nine pigs had functioning grafts, producing urine immediately post-transplant. Serum creatinine in these pigs followed a bell-shaped curve with peak values at D2-D3. Two renal artery

This is the version of the paper as accepted by "Transplant Proc".

This version has been through peer-review but still contains the authors' original formatting. It has not yet undergone the publisher's copy-editing and typesetting process, which will usually result in changes to the font and text alignment. 
thromboses were observed and the venous anastomosis was prone to stenosis in six cases. These technical complications influenced post-transplant observations. Renal artery thrombosis resulted in anuria and increasing levels of serum creatinine. A stenotic renal vein was associated with a higher peak of serum creatinine. Modifying the surgical technique accordingly, prevented these complications.

Conclusion. We developed and optimized a reproducible model of porcine kidney autotransplantation. Herein we demonstrated the importance of a proper surgical anastomotic technique to avoid inflow or outflow obstruction that might jeopardize post-transplant kidney function. This clinically relevant model offers the possibility to study various aspects related to kidney preservation, without interference of allogeneic immune responses. publisher's copy-editing and typesetting process, which will usually result in changes to the font and text alignment. 


\section{REPRODUCIBLE MODEL FOR KIDNEY AUTOTRANSPLANTATION IN PIGS}

\section{INTRODUCTION}

Kidney transplantation is the preferred treatment of end-stage renal disease and further application is currently hampered by an ongoing organ shortage. Now more than ever, research into the mechanisms of Warm Ischemia (WI), Cold Ischemia (CI), and Ischemia-Reperfusion Injury (IRI) is needed to maximize the use of the available donor pool and to minimize Primary Non-Function (PNF) and Delayed Graft Function (DGF). The majority of this research has been performed in small animal models, but extrapolating data obtained in rodents to humans is difficult. ${ }^{1}$ Preferably, the response of the kidney to WI, CI, and IRI needs to be studied in a model closer to humans. The pig seems to be the ideal species to serve this purpose. ${ }^{1}$ Several porcine models to study mechanisms of WI, CI and IRI exist: reperfusion models ${ }^{2,3}$, isolated organ preservation systems $s^{4,5}$ and kidney transplantation models. ${ }^{6-9}$ However, the technical and surgical aspects of these models and their possible pitfalls have not been described in detail. Therefore, the goal of the present study was to develop a porcine model for kidney autotransplantation, to report the difficulties encountered and to propose solutions to prevent these difficulties. This model can easily be modified into a model of kidney transplantation from non-heart-beating donors by exposing the kidney to WI prior to CI.

\section{METHODS}

\section{Animals}

Twelve female pigs (TOPIGS20, VOF van Beek, Lelystad, The Netherlands), weighing 24-51 kg, were allowed to become accustomed to their new environment for at least two days after transportation. They were housed in a conventional, closed housing system. They received a maintenance diet (MPig-H, ssniff, Soest, Germany) and had unlimited access to tap water. After surgery, the pigs were kept in single pens and had visual or auditory contact with each other. Experiments were performed in accordance with international guidelines. ${ }^{10}$ The study protocol was approved by the Animal Care Committee of the Catholic University of Leuven, Belgium. 


\section{Surgical procedures}

Surgical procedures were performed under sterile conditions. Food was withheld 12 hours before surgery. The procurement procedure at day -1 (D-1) started with the tranquilization of the pig by intramuscular injection of xylazine $(2 \mathrm{mg} / \mathrm{kg})$ and tiletamine/zolazepam $(8 \mathrm{mg} / \mathrm{kg})$ allowing endotracheal intubation and mechanical ventilation (Isoflurane 1\% in a mixture of $40 \%$ oxygen and room air) 10 minutes later. We used the following ventilation settings: tidal volume of $10-15 \mathrm{~mL} / \mathrm{kg}$, peak inspiratory pressure below $25 \mathrm{~cm} \mathrm{H}_{2} \mathrm{O}$ and an adjusted ventilation rate to keep the end tidal $\mathrm{CO}_{2}$ between 35-40 mmHg. Then, an ear vein was cannulated allowing intravenous administration of antibiotics (cefazoline $2 \mathrm{~g}$ ), followed by a continuous drip of fentanyl $(8 \mu \mathrm{g} / \mathrm{kg} / \mathrm{h})$ and a total of 40 $\mathrm{mL} / \mathrm{kg}$ of a balanced electrolyte solution (Plasma-Lyte $\mathrm{A}^{\circledR}$, Baxter, Brussels, Belgium). We monitored electrocardiogram, oxygen saturation and rectal temperature throughout the procedure and prevented hypothermia by heating the air around the pig to a temperature of $38^{\circ} \mathrm{C}$ using a Bair Hugger ${ }^{\circledR}$ (Augustine Medical Inc, Eden Prairi, MN, USA).

Next, we exposed and isolated the left carotid artery and left external jugular vein and inserted a single-lumen 16G catheter (Cavafix Certo $^{\circledR}$, Braun, Melsungen, Germany) in both vessels. The catheters were tunneled through the skin, allowing monitoring of arterial and central venous pressures and perioperative blood sampling.

We then performed a midline laparotomy and surgically inserted a bladder catheter. This catheter was tunneled through the skin and connected to an external collecting bag to allow postoperative monitoring of urine production. Finally, we retrieved the left kidney and the ureter by atraumatically dissecting them free from the retroperitoneum, leaving the blood supply to the ureter intact. The renal vascular pedicle of the graft was left as long as possible by freeing the renal artery and vein up to the aorta and the vena cava. The renal artery was cannulated immediately after nephrectomy and flushed with $500 \mathrm{~mL}$ of cold $\left(4^{\circ} \mathrm{C}\right)$ histidine-tryptophan-ketoglutarate preservation solution, and infused at a hydrostatic pressure of $100 \mathrm{~cm} \mathrm{H}_{2} \mathrm{O}$. The kidney was preserved in $500 \mathrm{~mL}$ of University of Wisconsin 
preservation solution at $4-6^{\circ} \mathrm{C}$ for 18 hours. The abdomen was closed and the administration of anesthetics stopped. After weaning the animal from the ventilator, it was returned to the pen.

The next day, the same animal was anesthetized in the same way as previously described. We then performed a right nephrectomy, dividing the renal artery and vein in the hilum and leaving the recipient renal vessels as long as possible to facilitate the autotransplantation of the left kidney. Transplantation was performed by end-to-end anastomoses between graft and recipient renal vessels, using 6.0 polypropylene sutures (Prolene ${ }^{\circledR}$, Ethicon, Belgium) for the artery and 6.0 or 7.0 Prolene $^{\circledR}$ for the vein. Throughout the procedure, attention was given to maintain the mean arterial pressure above $80 \mathrm{mmHg}$ and the central venous pressure above $10 \mathrm{mmHg}$. This was achieved by the administration of Geloplasma $^{\circledR}$ (Fresenius Kabi, Belgium) when necessary. During the arterial anastomosis, $100 \mathrm{~mL}$ of mannitol 20\% was given. Finally, an end-to-end uretero-ureterostomy over a 6F double-J stent (Ref. 5201800, Gyrus AMCI, Germany) was performed using Prolene ${ }^{\circledR} 6.0$.

\section{Postoperative course}

Immediately post-transplant, the animals had free access to food and water. Analgesia was given until 60 hours post-transplant (buprenorphine $0.30 \mathrm{mg} / \mathrm{kg} / 12 \mathrm{~h}$ intravenously) and crystalloid fluids (PlasmaLyte $\mathrm{A}^{\circledR} 20 \mathrm{~mL} / \mathrm{kg} / \mathrm{day}$ ) were administered until 36 hours post-transplant. Ten days post-transplant, the pigs were anesthetized and a re-laparotomy was performed for evaluation of the anastomoses and the macroscopic aspect of the kidney. At the end of this procedure, the animals were sacrificed by an intravenous injection of $20 \mathrm{~mL}$ of potassium chloride $14.9 \%$.

Peri- and post-operative monitoring

Blood samples were taken from the venous catheter on a daily basis for assessment of renal function by measuring serum creatinine. Arterial and venous catheters were flushed daily and filled with heparinized saline $(10 \mathrm{IU} / \mathrm{mL})$ to prevent obstruction of the catheters by blood clots. The macroscopic appearance of the kidney was documented after the flush, 15 and 45 minutes after reperfusion and at sacrifice. Urine production was monitored daily. Graft failure was defined as anuria.

\section{Experimental design}


The study was designed in two phases. In phase 1 , the surgical procedure and the arterial anastomotic technique were tested in four pigs after 24 hours CI. For the arterial anastomosis, a running Prolene ${ }^{\circledR}$ 6.0 was used. To avoid arterial thrombosis, the size of this anastomosis was enlarged by creating a Carrel-patch between the upper and lower polar artery on the recipient side (Figure 1). Prior to finishing this anastomosis, the recipient renal artery was flushed with heparinized saline $(10 \mathrm{IU} / \mathrm{mL})$ to avoid formation of a clot proximal to the vascular clamp (Figure 1). For the venous anastomosis, a Prolene ${ }^{\circledR} 6.0$ running suture was used but this caused a stenosis in three out of four anastomoses (Table 1). In this phase 1, the follow-up was limited and the timing of sacrifice was predetermined (D3, D5, D5, and D7). Presence or absence of urine production was noted and graft failure was defined as anuria.

In phase 2, we autotransplanted seven pig kidneys after 18 hours of CI. The arterial anastomosis technique was the same as in phase 1 . For the venous anastomosis, we paid special attention to avoid stenosis. For this purpose, we first switched from a Prolene ${ }^{\circledR} 6.0$ running suture to a Prolene ${ }^{\circledR} 7.0$ running suture. However, this still caused venous stenosis in the first three anastomoses of phase 2 (Table 1). Consequently, two stay sutures were added at each side of the anastomosis $\left(180^{\circ}\right)$ to perform the anastomosis under continuous traction, and the running suture was tied with a growth factor (Figure 1). Timing of sacrifice was predetermined (D10). Again, presence or absence of urine production was noted and graft failure was defined as anuria.

\section{RESULTS}

The outcome after kidney autotransplantation is represented in table 1. Four pigs were sacrificed before the end of the experiment. One animal developed therapy resistant pulmonary edema and was sacrificed before the end of the donor procedure. Two pigs developed symptoms of brain ischemia after transplantation that seemed to be related to flushing of the arterial catheter and were sacrificed at D1 and D3. On autopsy the latter of these pigs was found to have developed a thrombosis of a very small lower polar artery, but she produced urine. One pig was sacrificed at D9 instead of D10 for logistical reasons. 
During phase 1, two out of four pigs developed graft failure, but survived the predetermined follow-up period. Graft failure was caused by thrombosis of the renal artery. In phase 2 seven transplantations were performed. After three experiments in phase 2, our venous anastomotic technique had evolved to create a stenosis-free anastomosis.

All surviving pigs in phase 2 displayed graft function (urine production). Evolution of serum creatinine in recipients of failing grafts (anuria) versus functioning grafts (urine production) and in recipients of a functioning graft with a stenotic versus a non-stenotic venous anastomosis is represented in Figure 2. In functioning grafts, serum creatinine increased post-transplant, peaked at D2-D3 and then slowly decreased, almost reaching the level of serum creatinine after removal of the left kidney at D0. In contrast, in recipients with graft failure there was no urine production and serum creatinine continued to increase.

\section{DISCUSSION}

We established a reproducible porcine model for kidney autotransplantation to study preservationrelated issues. To avoid allogeneic immune response and rejection we developed an autotransplant model. To avoid urine production from native kidneys and to allow following graft function by measurement of creatinine, we removed both native kidneys. We elected a standard CI time of 18 hours according to international clinical practice and species specific guidelines. Indeed, Opelz et al reported a mean CI time of 16.6 hours in a multicenter analysis of human kidney preservation. ${ }^{11}$ Nicholson et al. recommended to limit CI time in porcine kidney transplantation to 18-20 hours to avoid PNF and DGF, which were found to be related to the length of CI. ${ }^{6}$ A CI time of 18 hours also allows performing the donor and recipient surgery during the day.

We preferred female over male pigs for anatomical reasons. The urethra of male pigs is located superficially at the midline, hampering the performance of a midline laparotomy. However, by choosing female pigs, there is a risk for bias since the female kidney is found to be more resistant to IRI. ${ }^{12}$ This resistance of female organs to IRI is thought to be caused by sex hormones, which are believed to contribute to the pathophysiology of IRI through regulation of inflammation. ${ }^{12}$ To avoid 
this possible bias, we opted to use prepubescent pigs. On average, puberty in female pigs starts at about 6-7 months ${ }^{13}$; we used 3-4 month old pigs, weighing around $35-45 \mathrm{~kg}$. At this weight, the diameter of the renal vessels is large enough to allow adequate vascular anastomoses.

Nevertheless, our anastomotic technique needed to be adjusted twice. Two animals in phase 1 developed a thrombosis of the renal artery. To enlarge the size of the anastomosis and thus reduce the risk of thrombosis, we created a Carrel-patch between the upper and lower polar artery on the recipient side (Figure 1). In one case however, the arterial thrombosis was not related to the anastomotic technique, but was caused by a blood clot formed just proximally to the vascular clamp placed on the native renal artery (Figure 1). Hypercoagulability in pigs, believed to be caused by increased activity of Factors V-VIII-IX-XII ${ }^{14}$, most likely contributed to this problem. Because no heparin is given during transplantation, we started releasing the arterial clamp prior to finishing the anastomosis to make sure that any clot would be flushed out. This was followed by flushing the native renal artery with heparinized saline $(10 \mathrm{IU} / \mathrm{mL})$, replacing the clamp and finishing the anastomosis. During this flush, the artery on the recipient side was closed with a bulldog-clamp to avoid any reperfusion taking place before releasing the venous and arterial clamps. After the aforementioned adjustments, all grafts were functional at the time of sacrifice. We only encountered one additional arterial problem where a thrombosis of the lower polar artery occurred, probably due to its very small size. A second technical improvement was necessary to avoid stenosis of the venous anastomosis. Because of the extremely thin wall of the porcine renal vein, such a stenosis can easily arise and this problem occurred in three out of four experiments in phase 1. To avoid formation of venous stenosis, we first switched from Prolene ${ }^{\circledR} 6.0$ to 7.0 in the first three experiments of phase 2 . However, with unsatisfactory results. Consequently, two stay sutures were placed and kept under continuous traction during the entire anastomosis and - in addition - the suture was tied with a growth factor (Figure 1). By doing this, a stenosis-free anastomosis was created in the last four experiments. Although no grafts with a venous stenosis showed macroscopic signs of venous congestion after reperfusion and all produced urine, the serum creatinine levels post-transplant were higher compared to grafts with a non-stenotic vein (Figure 2). This clearly indicates the importance of a proper surgical technique to make sure that the 
evolution of post-transplant creatinine reflects the biological behavior of the graft and is not influenced by technical factors such as inflow or outflow obstruction.

Initially we were concerned that two anesthetic procedures within 24 hours would be tolerated badly and that e.g. ileus would occur due to the manipulation of the intestines. However, all pigs woke up quickly after cessation of anesthesia and could easily be weaned from the ventilator. With adequate analgesia, all were ambulant the evening after transplantation, and no ileus or infections occurred.

Another unexpected problem that we observed was related to the arterial catheters. In two pigs we witnessed the occurrence of non-recovering brain ischemia (Table 1). Immediately after flushing the arterial catheter with $10 \mathrm{~mL}$ of heparinized saline $(10 \mathrm{IU} / \mathrm{mL})$, both animals dropped to their side and were unable to move. One pig started to vomit, the other developed epileptic attacks every two minutes. Both animals were sacrificed. These symptoms could be caused by the mobilization of a clot within the catheter or possibly by an air embolism. However, during all our experiments, care was taken to avoid air entering the catheters, to flush them with heparinized saline daily and to position them correctly. Because we did not observe any blood clots or other problems during the autopsies, we hypothesized that these symptoms might be related directly to the flushing of the arterial catheters with an exceeding amount $(10 \mathrm{~mL})$ of heparinized saline in the right (unique) carotid artery. Since the common carotid artery of pigs has its origin on the aortic arch and bifurcates into the left and right carotid arteries, the left carotid artery can be tied off in the presence of a functioning circle of Willis. ${ }^{15}$ It can be imagined that by the instillation of a relatively large volume of saline through the arterial catheter, the blood in the common carotid artery and subsequently in the circle of Willis is replaced by saline, causing transient oxygen shortage in the left hemisphere leading to symptoms of brain ischemia. We consequently reduced the volume of the flush solution to $1.5 \mathrm{~mL}$ and no more cerebral complications were observed.

To follow the renal function of the transplanted kidney, we determined serum creatinine levels. Serum creatinine is regarded as an unreliable indicator during acute kidney injury. ${ }^{16}$ From our results, it appears that serum creatinine is also unreliable in the detection of early graft failure. Figure 2 shows a similar initial rise in serum creatinine between non-functioning and functioning grafts. Only after 
functioning grafts reached their serum creatinine peak at D2-D3, a distinction could be made between functioning and failing grafts.

In conclusion, we developed a reproducible and clinically relevant porcine kidney autotransplantation model. This model offers the possibility to study various aspects related to kidney preservation in general, without interference of allogeneic immune responses. By clamping the renal artery and vein during the donor procedure for a given period of time before cold flushing, this model can easily be modified to serve as a non-heart-beating model for kidney transplantation. This will allow studying the tolerance to- and the mechanisms of WI, CI and IRI in a true transplant setting.

\section{ACKNOWLEDGMENTS}

Mr. B. Theunis provided excellent surgical assistance.

Special gratitude goes to MG. Snoeijs, LW van Heurn and GW Schurink from the Department of Surgery, University Hospital Maastricht, Maastricht, The Netherlands for sharing their experience. 


\section{References}

1. Lieberthal W, Nigam SK: Acute renal failure. II. Experimental models of acute renal failure: imperfect but indispensable. Am J Physiol Renal Physiol 278:F1, 2000

2. Doucet C, Zhang K, Desurmont T, et al: Influence of warm ischemia time on peripheral-type benzodiazepine receptor: a new aspect of the role of mitochondria. Nephron Exp Nephrol 107:e1, 2007

3. Orvieto MA, Tolhurst SR, Chuang MS, et al: Defining maximal renal tolerance to warm ischemia in porcine laparoscopic and open surgery model. Urology 66:1111, 2005

4. Bagul A, Hosgood SA, Kaushik M, et al: Effects of erythropoietin on ischaemia/reperfusion injury in a controlled nonheart beating donor kidney model. Transpl Int 21:495, 2008

5. Gok MA, Shenton BK, Peaston R, et al: Use of streptokinase in a non-heart-beating donor animal model. Transplant Proc 34:2615, 2002

6. Nicholson ML, Hosgood SA, Metcalfe MS, et al: A comparison of renal preservation by cold storage and machine perfusion using a porcine autotransplant model. Transplantation 78:333, 2004

7. Treckmann JW, Paul A, Saad S, et al: Primary organ function of warm ischaemically damaged porcine kidneys after retrograde oxygen persufflation. Nephrol Dial Transplant 21:1803, 2006

8. Doucet C, Milin S, Favreau F, et al: A p38 mitogen-activated protein kinase inhibitor protects against renal damage in a non-heart-beating donor model. Am J Physiol Renal Physiol 295:F179, 2008 9. Maathuis $\mathrm{MH}$, Manekeller S, van der Plaats A, et al: Improved kidney graft function after preservation using a novel hypothermic machine perfusion device. Ann Surg 246:982, 2007

10. Commission of the European Communities. Guidelines for the accommodation and care of animals used for experimental and other scientific purposes; 2007/526/EC.

11. Opelz G, Dohler B: Multicenter analysis of kidney preservation. Transplantation 83:247, 2007

12. Park KM, Kim JI, Ahn Y, et al: Testosterone is responsible for enhanced susceptibility of males to ischemic renal injury. J Biol Chem 279:52282, 2004

13. Tummaruk P, Lundeheim N, Einarsson S, et al: Factors influencing age at first mating in purebred Swedish Landrace and Swedish Yorkshire gilts. Anim Reprod Sci 63:241, 2000 
14. Roussi J, Andre P, Samama M, et al: Platelet functions and haemostasis parameters in pigs: absence of side effects of a procedure of general anaesthesia. Thromb Res 81:297, 1996

15. Burbridge B, Matte G, Remedios A: Complex intracranial arterial anatomy in swine is unsuitable for cerebral infarction projects. Can Assoc Radiol J 55:326, 2004

16. Bellomo R, Kellum JA, Ronco C: Defining acute renal failure: physiological principles. Intensive Care Med 30:33, 2004 


\section{LEGENDS OF FIGURES AND TABLES}

Table 1. Outcome after kidney autotransplantation in pigs: phase 1: development of surgical procedure; phase 2: experimental protocol

Figure 1 Illustration of the optimized anastomotic technique as used in our porcine model of kidney autotransplantation

Figure 2 Mean serum creatinine levels after porcine kidney autotransplantation in recipients with graft failure (anuria) versus graft function (urine production) and in recipients with a functioning graft with a stenotic versus non-stenotic venous anastomosis

This is the version of the paper as accepted by "Transplant Proc".

This version has been through peer-review but still contains the authors' original formatting. It has not yet undergone the publisher's copy-editing and typesetting process, which will usually result in changes to the font and text alignment. 
Table 1

\begin{tabular}{|c|c|c|c|c|c|c|}
\hline & CI (h) & $\begin{array}{l}\text { Urine } \\
\text { production }\end{array}$ & Sacrifice & $\begin{array}{l}\text { Arterial } \\
\text { thrombosis }\end{array}$ & $\begin{array}{l}\text { Stenotic } \\
\text { vein }\end{array}$ & Remarks \\
\hline \multicolumn{7}{|c|}{ Phase 1 } \\
\hline 1 & $23: 45$ & + & D5 & - & + & \\
\hline 2 & $20: 58$ & - & D5 & + & + & \\
\hline 3 & $25: 33$ & - & D7 & + & + & \\
\hline 4 & $\begin{array}{c}22: 05 \\
\text { mean 23:25 }\end{array}$ & + & D3 & - & - & \\
\hline \multicolumn{7}{|c|}{ Phase 2} \\
\hline 5 & $18: 57$ & + & D10 & - & + & 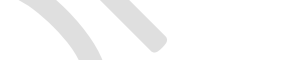 \\
\hline 6 & $18: 38$ & + & D10 & - & & \\
\hline 7 & $18: 25$ & + & D3 & $\begin{array}{l}\text { Lower } \\
\text { polar artery }\end{array}$ & & $\begin{array}{l}\text { Brain ischemia; } \\
\text { neurologic deficit }\end{array}$ \\
\hline 8 & $18: 31$ & + & D1 & & & $\begin{array}{l}\text { Brain ischemia; } \\
\text { neurologic deficit }\end{array}$ \\
\hline 9 & $17: 48$ & + & D9 & & - & \\
\hline 10 & $18: 28$ & + & D10 & - & - & \\
\hline 11 & NA & NA & D-1 & NA & NA & Pulmonary edema \\
\hline 12 & $\begin{array}{c}17: 58 \\
\text { mean } 18: 23\end{array}$ & + & D10 & - & - & \\
\hline
\end{tabular}

CI: cold ischemia in hours; D: postoperative day; NA: not applicable

This is the version of the paper as accepted by "Transplant Proc".

This version has been through peer-review but still contains the authors' original formatting. It has not yet undergone the publisher's copy-editing and typesetting process, which will usually result in changes to the font and text alignment. 
Figure 1

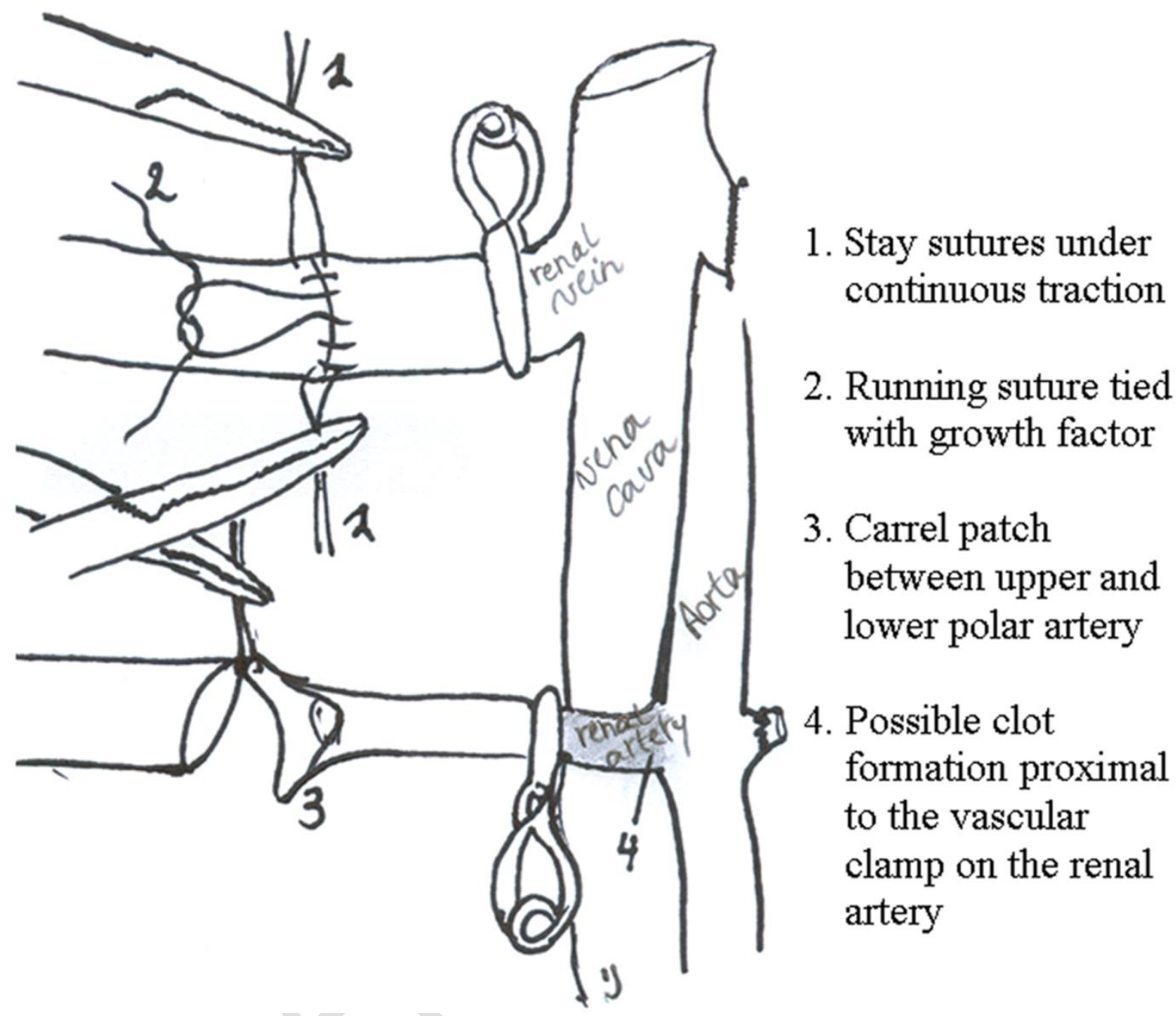

This is the version of the paper as accepted by "Transplant Proc".

This version has been through peer-review but still contains the authors' original formatting. It has not yet undergone the publisher's copy-editing and typesetting process, which will usually result in changes to the font and text alignment. 
Figure 2

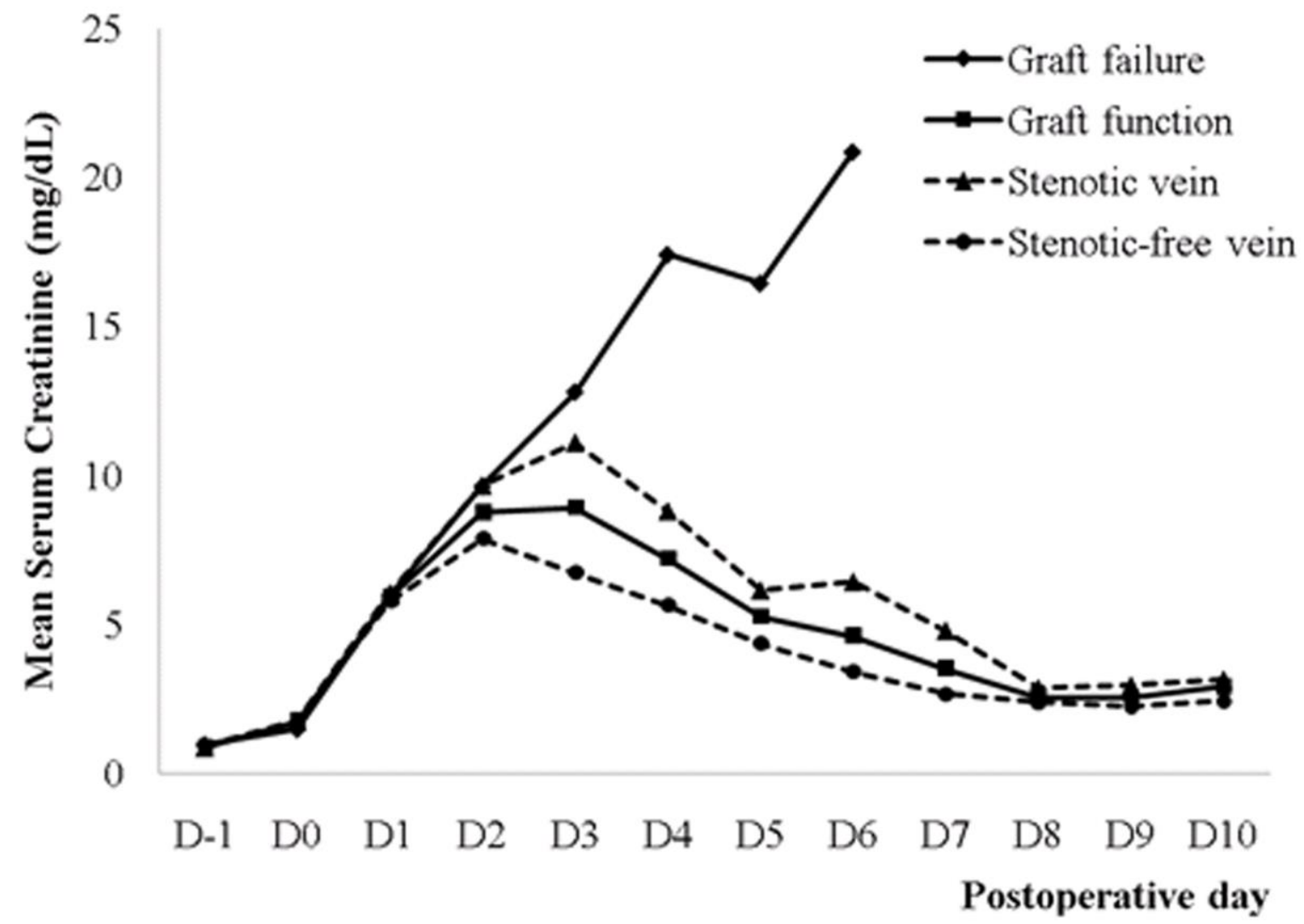

This is the version of the paper as accepted by "Transplant Proc".

This version has been through peer-review but still contains the authors' original formatting. It has not yet undergone the publisher's copy-editing and typesetting process, which will usually result in changes to the font and text alignment. 